\title{
Is Gut Microbial LPS a Potential Trigger of Juvenile Idiopathic Arthritis?
}

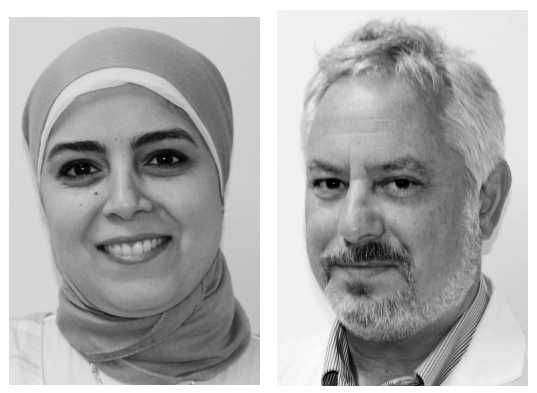

Juvenile idiopathic arthritis (JIA) is one of the most common chronic inflammatory disorders in children, with hallmarks of joint inflammation, synovial hyperplasia, and leukocyte infiltration ${ }^{1}$. As in other autoimmune diseases, the exact etiopathogenesis, and pathogenic drivers of JIA, remain incompletely understood. In rheumatoid arthritis (RA) and ankylosing spondylitis (AS), several studies have shown that disease susceptibility is strongly associated with sets of immune-response genes, which include specific MHC alleles. In contrast, JIA does not seem to have such a strong association, and there is less occurrence of familial aggregations, and only limited disease concordance in homozygotic twins s, $^{2,4,5}$.

All of these observations suggest a key role in JIA pathogenesis for noninherited factors. Indeed, a number of reports have implicated maternal factors, such as breast-feeding, and whether the mother is a smoker ${ }^{6}$. Other environmental factors have been cited, such as bacterial or viral infection, antibiotic usage $^{7}$, and most recently the influence of shifts in the gut microbiome ${ }^{8}$. The development of state-of-the-art 16S rRNA high-throughput sequencing technology for microbial profiling has opened the door for the characterization of the phylogenetic distribution of taxa in the gut bacterial communities in an individual. With this culture-independent approach, an increasing number of reports have documented decreases in gut microbiome diversity and other forms of dysbiosis in inflammatory and autoimmune diseases that include $\mathrm{RA}^{9,10,11}$, psoriatic arthritis ${ }^{12}, \mathrm{AS}^{13}$, and inflammatory bowel disease (IBD) ${ }^{14}$.

Primary epithelial barriers are the first line of immune defense, and an intact gut intestinal mucosa barrier controls the rate of exchange of nutritional elements from the gut lumen after processing by microbial pathways. Perturbations in barrier integrity can be triggered by poor nutrition, infection, local inflammatory factors, or certain medications, which can all contribute to intestinal permeability or a "leaky gut." Increased intestinal permeability can lead to the translocation of proinflammatory mediators and even entire bacteria into the lamina propria, a condition associated with diverse gastrointestinal inflammatory disorders including IBD, and especially celiac disease ${ }^{15}$. Moreover, increases in intestinal permeability may lead to the translocation of bacterial antigens into the circulation and other tissues; the bacterial DNA and peptidoglycans have been documented in the synovial linings of patients with active RA ${ }^{15}$.

Increases in intestinal permeability have also been reported in children with JIA - it appears to be a common consequence of microscopic colitis ${ }^{16,17,18}$. Lipopolysaccharide (LPS), a potent immune-activating factor from many aerobic gram-negative rods, is known to activate innate cells such as neutrophils and macrophages. This action induces secretion of proinflammatory cytokines and can result in joint inflammation ${ }^{19}$.

In this edition of The Journal, Fotis, et al ${ }^{20}$ investigated the hypothesis that the translocation of LPS from the bacterial community in the gut lumen into the circulation may contribute to the induction of systemic immune responses and the joint inflammation of patients with JIA. Patients enrolled in this study had new onset JIA without prior systemic therapy, and at the time of evaluation had either active polyarticular or oligoarticular JIA. As disease controls, the authors also studied samples from patients with spondyloarthropathies (SpA), including new-onset IBD with overlap RA, and from healthy individuals. These investigators used complementary approaches and documented that, compared to healthy controls, there were high IgG antiLPS antibody levels, as well as raised LPS binding protein (LBP) and $\alpha$-1-acid glycoprotein ( $\alpha$-1AGP) in serum samples of patients with JIA or SpA. Additionally, the raised levels of LBP and $\alpha-1$ AGP, a known immune modulatory agent, were also strongly correlated with C-reactive protein concentrations, a marker of systemic inflammation and with the disease activity score, while levels of anti-LPS IgG antibodies had weaker correlations. These findings provide further evidence for the hypothesis that, because of the translocation of gut bacterial components as a consequence

See Gut-driven inflammation in JIA, page 1624

Personal non-commercial use only. The Journal of Rheumatology Copyright (C) 2017. All rights reserved. 
of gut permeability, shifts in the gut microbiome may contribute to JIA pathogenesis.

The authors argue that the $\alpha-1$ AGP test may be a useful prognostic tool for predicting JIA prognosis. This test is certainly not disease-specific; therefore further studies are needed to determine its utility in other inflammatory diseases, especially in conditions with pathogenic pathways common to JIA. In general, the Fotis, et al studies highlight the importance of dynamic balance and homeostasis in the gut microbiota, and suggest that altered internal environmental factors may drive inflammatory joint diseases and adversely affect quality of life.

From a broader perspective, these studies contribute to a growing literature that suggests that disturbances in the complex communities residing in the human gut correlate with, and may directly contribute to, the pathogenesis of a range of rheumatic conditions. While these correlative studies have not quite elucidated the suspected causative disease drivers, we need to better understand the basis for these dysbioses and the associated postulated expansions of pathobionts and/or contractions of protector microbial species. We are also beginning to wonder whether past antibiotic exposures can also be responsible. More importantly, if gut dysbioses are key pathogenic drivers in JIA, research programs are needed to design the best interventional approaches for restoring balanced intestinal communities.

All of these factors add new dimensions to the hygiene hypothesis, which postulates that the reduced exposure to microorganisms in industrialized countries, owing to improved sanitary conditions and antibiotic exposure, reduces the complexity of our microbiomes and skews them, directly or indirectly promoting the development of allergic and autoimmune diseases ${ }^{21}$.

In this context, the health of a child may be affected by fundamental influences dating to the earliest time of intestinal colonization and community establishment in the gut. Indeed, we have only recently appreciated the obvious fact that breast-feeding generally provides the best, evolutionarily selected nutritional source for the neonate, because breast milk contains a variety of immune-modulating compounds, both immune cells and their products such as cytokines. Indeed, the first breast milk, colostrum, contains high levels of secretory IgA antibody, which equals that found in the bloodstream of adults ${ }^{22}$. Breast-feeding may, therefore, result in very beneficial immunological imprinting of the infant. In support, a recent study from Sweden showed that breastfeeding for more than 4 continuous months correlated with protection from the later development of JIA ${ }^{23}$. Further, an independent study from a French group similarly showed the protective effect of breast-feeding in a case-control retrospective study of patients with $\mathrm{AS}^{24}$.

In addition to the current report from Fotis, et $\mathrm{al}^{20}$, there is mounting evidence of the central roles of mucosal immunity in homeostasis, and that specific microbial factors may play central roles in the pathogenesis of pediatric inflammatory arthritis. Moreover, the seeds of these conditions may have been planted at the time of postnatal intestinal colonization or may reflect the influence of antibiotic regimens that may not always be needed.

\section{ACKNOWLEDGMENT}

With great pleasure we acknowledge the Judith and Stewart Colton Foundation and its support for our research work.

\section{DOUA F. AZZOUZ, $\mathrm{PhD}$ GREGG J. SILVERMAN, MD,}

Laboratory of B cell Immunobiology, Department of Medicine,

New York University School of Medicine, New York, New York, USA.

Address correspondence to Dr. G.J. Silverman, Laboratory of B cell Immunobiology, Department of Medicine, New York University School of Medicine, Room 804, 450 E. 29th St., New York, New York 10016, USA. E-mail: Gregg.silverman@nyumc.org

\section{REFERENCES}

1. Petty RE. Growing pains: the ILAR classification of juvenile idiopathic arthritis. J Rheumatol 2001;28:927-8.

2. Cobb JE, Hinks A, Thomson W. The genetics of juvenile idiopathic arthritis: current understanding and future prospects. Rheumatology 2014;53:592-9.

3. Okada Y, Wu D, Trynka G, Raj T, Terao C, Ikari K, et al. Genetics of rheumatoid arthritis contributes to biology and drug discovery. Nature 2014;506:376-81.

4. Prahalad S, Zeft AS, Pimentel R, Clifford B, McNally B, Mineau GP, et al. Quantification of the familial contribution to juvenile idiopathic arthritis. Arthritis Rheum 2010;62:2525-9.

5. Savolainen A, Saila H, Kotaniemi K, Kaipianen-Seppanen O, Leirisalo-Repo M, Aho K. Magnitude of the genetic component in juvenile idiopathic arthritis. Ann Rheum Dis 2000;59:1001

6. Ellis JA, Munro JE, Ponsonby AL. Possible environmental determinants of juvenile idiopathic arthritis. Rheumatology 2010;49:411-25.

7. Arvonen M, Virta LJ, Pokka T, Kroger L, Vahasalo P. Repeated exposure to antibiotics in infancy: a predisposing factor for juvenile idiopathic arthritis or a sign of this group's greater susceptibility to infections? J Rheumatol 2015;42:521-6.

8. Arvonen M, Berntson L, Pokka T, Karttunen TJ, Vahasalo P, Stoll ML. Gut microbiota-host interactions and juvenile idiopathic arthritis. Pediatr Rheumatol Online J 2016;14:44.

9. Scher JU, Sczesnak A, Longman RS, Segata N, Ubeda C, Bielski C, et al. Expansion of intestinal Prevotella copri correlates with enhanced susceptibility to arthritis. Elife 2013;2:e01202.

10. Severijnen AJ, van Kleef R, Hazenberg MP, van de Merwe JP. Cell wall fragments from major residents of the human intestinal flora induce chronic arthritis in rats. J Rheumatol 1989;16:1061-8.

11. Zhang X, Rimpilainen M, Simelyte E, Toivanen P. Characterisation of Eubacterium cell wall: peptidoglycan structure determines arthritogenicity. Ann Rheum Dis 2001;60:269-74.

12. Scher JU, Ubeda C, Artacho A, Attur M, Isaac S, Reddy SM, et al. Decreased bacterial diversity characterizes the altered gut microbiota in patients with psoriatic arthritis, resembling dysbiosis in inflammatory bowel disease. Arthritis Rheumatol 2015; 67:128-39.

13. Rosenbaum JT, Lin P, Asquith M, Costello ME, Kenna TJ, Brown MA. Does the microbiome play a causal role in spondyloarthritis? Clin Rheumatol 2014;33:763-7.

Personal non-commercial use only. The Journal of Rheumatology Copyright $\subset$ $\subset$ 2017. All rights reserved 
14. Sokol H, Seksik P, Rigottier-Gois L, Lay C, Lepage P, Podglajen I, et al. Specificities of the fecal microbiota in inflammatory bowel disease. Inflamm Bowel Dis 2006;12:106-11.

15. Bischoff SC, Barbara G, Buurman W, Ockhuizen T, Schulzke JD, Serino $\mathrm{M}$, et al. Intestinal permeability - a new target for disease prevention and therapy. BMC Gastroenterol 2014;14:189.

16. Picco P, Gattorno M, Marchese N, Vignola S, Sormani MP, Barabino A, et al. Increased gut permeability in juvenile chronic arthritides. A multivariate analysis of the diagnostic parameters. Clin Exp Rheumatol 2000;18:773-8.

17. Pichler J, Ong C, Shah N, Sebire N, Kiparrissi F, Borrelli O, et al. Histopathological features of gastrointestinal mucosal biopsies in children with juvenile idiopathic arthritis. Pediatr Res 2016; 79:895-901.

18. Weber P, Brune T, Ganser G, Zimmer KP. Gastrointestinal symptoms and permeability in patients with juvenile idiopathic arthritis. Clin Exp Rheumatol 2003;21:657-62.

19. Lorenz W, Buhrmann C, Mobasheri A, Lueders C, Shakibaei M. Bacterial lipopolysaccharides form procollagen-endotoxin complexes that trigger cartilage inflammation and degeneration: implications for the development of rheumatoid arthritis. Arthritis Res Ther 2013;15:R111.

20. Fotis L, Shaikh N, Baszis KW, Samson CM, Lev-Tzion R, French AR, et al. Serological evidence of gut-driven systemic inflammation in juvenile idiopathic arthritis. J Rheumatol 2017;44:1624-31.

21. Okada H, Kuhn C, Feillet H, Bach JF. The "hygiene hypothesis" for autoimmune and allergic diseases: an update. Clin Exp Immunol 2010;160:1-9.

22. Hurley WL, Theil PK. Perspectives on immunoglobulins in colostrum and milk. Nutrients 2011;3:442-74.

23. Kindgren E, Fredrikson M, Ludvigsson J. Early feeding and risk of juvenile idiopathic arthritis: a case control study in a prospective birth cohort. Pediatr Rheumatol Online J 2017;15:46.

24. Montoya J, Matta NB, Suchon P, Guzian MC, Lambert NC, Mattei JP, et al. Patients with ankylosing spondylitis have been breast fed less often than healthy controls: a case-control retrospective study. Ann Rheum Dis 2016;75:879-82.

J Rheumatol 2017;44:1569-71; doi:10.3899/jrheum.170791 